\title{
Linear Programming Design of Coefficient Decimation FIR Filters
}

\author{
Zaka Ullah Sheikh and Oscar Gustafsson
}

\section{Linköping University Post Print}

N.B.: When citing this work, cite the original article.

(C2012 IEEE. Personal use of this material is permitted. However, permission to reprint/republish this material for advertising or promotional purposes or for creating new collective works for resale or redistribution to servers or lists, or to reuse any copyrighted component of this work in other works must be obtained from the IEEE.

Zaka Ullah Sheikh and Oscar Gustafsson, Linear Programming Design of Coefficient Decimation FIR Filters, 2012, IEEE Transactions on Circuits and Systems - II - Express Briefs, (59), 1, 60-64.

http://dx.doi.org/10.1109/TCSII.2011.2173965

Postprint available at: Linköping University Electronic Press

http://urn.kb.se/resolve?urn=urn:nbn:se:liu:diva-75082 


\title{
Linear Programming Design of Coefficient Decimation FIR Filters
}

\author{
Zaka Ullah Sheikh, Student Member, IEEE and Oscar Gustafsson, Senior Member, IEEE
}

\begin{abstract}
The coefficient decimation technique for reconfigurable FIR filters was recently proposed as a filter structure with low computational complexity. In this brief, we propose to design these filters using linear programming taking all configuration modes into account, instead of only consider the initial reconfiguration mode as in previous works. Minimax solutions with significantly lower approximation errors compared to the straightforward design method in earlier works are obtained. In addition, some new insights that are useful when designing coefficient decimation filters are provided.
\end{abstract}

Index Terms-FIR filters, Coefficient decimation, Reconfigurability, Linear programming, Approximation error

\section{INTRODUCTION}

$\mathbf{C}$ OEFFICIENT decimation is a technique to implement reconfigurable FIR filters [1]. The technique is based on decimating and/or interpolating a fixed set of impulse response coefficients. In this way, several different frequency responses are obtained without changing the coefficient values. Hence, there is no need to store different filter coefficient sets and techniques for simplifying the multipliers, such as shift-andadd techniques, can be straightforwardly applied.

Many architectures for different applications have been proposed based upon the coefficient decimation technique. These different architectures find their use in filter bank channelizers for multi-standard applications [2]-[4], spectrum sensing in cognitive radios [5], and channel adaptation in cognitive radios [6]. Most of these approaches operate with a scheme similar to frequency-response masking (FRM) filters [7], where the model filter is using the coefficient decimation technique, and, hence, can be reconfigured. Clearly, the masking filter(s) needs to be reconfigured accordingly, which is often solved by using several sets of filter coefficients. Still, the coefficient decimation technique can be used for reconfigurable single stage filters as well.

In the previous works [1], [2], [4]-[6], the design has been performed using the Parks-McClellan algorithm [8] only considered the initial filter specification (the model filter). Interpolating the impulse response results in a compression of the frequency response. However, decimating it will deteriorate

The authors are with the Division of Electronics Systems, Department of Electrical Engineering, Linköping University, SE-581 83 Linköping, Sweden, e-mail: \{zaka, oscarg\}@isy.liu.se.

The work of Z. U. Sheikh was supported by the Higher Education Commission, Pakistan. The work of O. Gustafsson was supported by a career contract from Linköping University.

Manuscript received March 6, 2011. Revised July 10, 2011 and Sept. 13, 2011.

Copyright (c) 2011 IEEE. Personal use of this material is permitted. However, permission to use this material for any other purposes must be obtained from the IEEE by sending an email to pubs-permissions@ieee.org the frequency response to possibly break the specifications. In this brief, we propose an offline design method based on linear programming that takes all required configuration modes into consideration and results in a minimax solution over all configurations. The resulting filter coefficients can replace the ones in previous work to either decrease the worst approximation error and/or decrease the filter order required for a given approximation error in the reconfiguration modes.

Following this introduction, Section II reviews the coefficient decimation technique. In Section III the proposed design approach is presented. In Section IV, two design examples are illustrating the properties of the proposed design method, whereas Section V concludes the brief.

\section{Coefficient Decimation Review}

The initial filter, called the model filter analogous with FRM filters, is assumed to be an even-order linear-phase lowpass FIR filter with passband edge $\omega_{c} T$ and stopband edge $\omega_{s} T$. The flexibility of the coefficient decimation technique is obtained by having two parameters defining how the impulse response of the model filter is modified: decimation by $D$ followed by interpolation by $M^{1}$. The decimation leads to that the number of multiplications is reduced by a factor of $D$. The magnitude response after decimation has a bandwidth and transition bandwidth $D$ times larger than that of the model filter. The interpolation results in a shrinking of bandwidth and transition bandwidth by a factor of $1 / M$ and a periodic magnitude response with a period of $2 \pi / M$. Hence, the decimation and interpolation factors, $D$ and $M$, effect the band edges as

$$
\omega_{c} T^{\prime}=\frac{D}{M} \omega_{c} T
$$

and

$$
\omega_{s} T^{\prime}=\frac{D}{M} \omega_{s} T
$$

where $\omega_{c} T^{\prime}$ and $\omega_{s} T^{\prime}$ are the passband and stopband edges of the resulting filter.

The different filter magnitude responses are shown in Fig. 1 which represents the model filter $H\left(e^{j \omega T}\right)$, decimated only version $H_{D, 1}\left(e^{j \omega T}\right)$, interpolated only version $H_{1, M}\left(e^{j \omega T}\right)$ and both decimated and interpolated version $H_{D, M}\left(e^{j \omega T}\right)$.

It can be noted that a coefficient decimation filter can result in $D_{\max } M_{\max }$ different configurations, where $D_{\max }$ and

\footnotetext{
${ }^{1}$ Interpolation with $M$ followed by decimation with $D$ results in exactly the same impulse response when $D$ and $M$ are relative prime [9]. If not, the impulse response is equivalent to decimation with $D^{\prime}$ followed by interpolation with $M^{\prime}$, where $D^{\prime}=D / G, M^{\prime}=M / G$, and $G=\operatorname{gcd}(D, M)$, i.e., the greatest common divisor of $D$ and $M$. This can be easily realized by noting that interpolation with $G$ followed by decimation with $G$ will leave a sequence unchanged.
} 
(a)

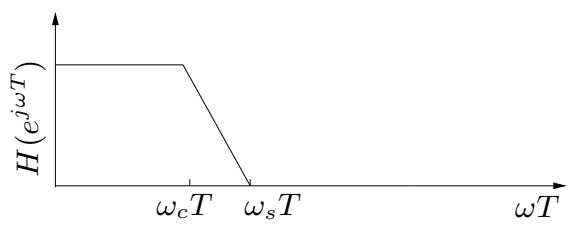

(b)

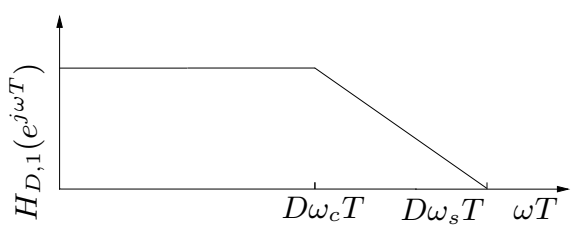

(c)

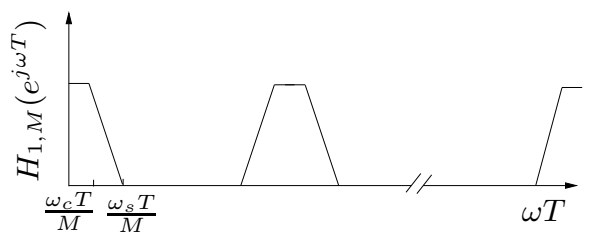

(d)

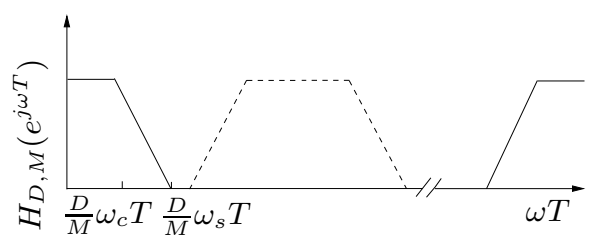

Fig. 1. Magnitude responses of different filters: (a) model filter, (b) decimated only model filter, (c) interpolated only model filter, and (d) decimated and interpolated model filter.

$M_{\max }$ represent the maximum possible value of the decimation and interpolation factors, respectively. A suitable value of $M_{\max }$ is selected on the basis of required configurations, while there is an upper limit on the value of $D_{\max }$. The upper limit on $D_{\max }$ can be found by

$$
D_{\max } \leq\left\lfloor\frac{\pi}{\omega_{s} T}\right\rfloor .
$$

While not formally required, it is often preferred to select the filter order, $N$, to be an integer multiple of the decimation factors, $D$.

Finally, it should be noted that for even $D$ there are two different ways to decimate an even-order impulse response to obtain a linear-phase filter [10]. To the best of the authors' knowledge, this has not been considered in any of the previous works on coefficient decimation filters. However, in this brief we provide some insight into this issue by designing one of the examples using all different combinations of decimation.

\section{Proposed Design Approach}

The proposed design, as discussed earlier, is based on application of linear programming on the coefficient decimation technique to obtain an optimal solution in the minimax $\left(H_{\infty}\right)$ sense. For ease of exposition we assume that the filter order $N$ is an even multiple of all decimation factors $D_{i}$.

\section{A. Review of Linear Programming FIR Filter Design}

In general, when designing linear-phase FIR filters, it is convenient to express their magnitude responses with the aid of a real function denoted $H_{R}(\omega T)$ as

$$
H\left(e^{j \omega T}\right)=e^{j \phi(\omega T)} H_{R}(\omega T) .
$$

The function $H_{R}(\omega T)$ is called the zero-phase frequency response of $H(z)$. Clearly, the magnitude response of $H\left(e^{j \omega T}\right)$ is equal to the magnitude of $H_{R}(\omega T)$, i.e.

$$
\left|H\left(e^{j \omega T}\right)\right|=\left|H_{R}(\omega T)\right| .
$$

In order to formulate the problems to be utilized in linear programming and for convenience we make use of the function trig which has the form $\operatorname{trig}(k, \omega T)$. It takes as input a scalar $k$ which corresponds to the filter tap value, numbered from the center tap $k=0,1,2, \ldots, K$, and the angle $\omega T$ at which the zero-phase frequency response is evaluated. For an evenorder symmetric FIR filter $K=N / 2$, while for an odd-order symmetric FIR filter $K=(N-1) / 2$ [9]. The zero-phase frequency response $H_{R}(\omega T)$ can be written with the help of $\operatorname{trig}(k, \omega T)$ and $h(k)$, i.e., $k$ :th filter coefficient from the center tap as

$$
H_{R}(\omega T)=\sum_{k=0}^{K} h(k) \operatorname{trig}(k, \omega T),
$$

where for $\operatorname{trig}(k, \omega T)$ we have

$$
\operatorname{trig}(k, \omega T)= \begin{cases}1, & k=0 \\ 2 \cos (\omega T k), & k \geq 1\end{cases}
$$

for an even-order symmetric FIR filter and

$$
\operatorname{trig}(k, \omega T)=2 \cos \left(\omega T\left[k-\frac{1}{2}\right]\right)
$$

for an odd-order symmetric FIR filter.

Let $\delta \geq 0$ represents the maximum approximation error, defined as

$$
\delta=\max _{\omega T}\left[W(\omega T)\left|H_{R}(\omega T)-A(\omega T)\right|\right],
$$

where $A(\omega T)$ is the desired amplitude and $W(\omega T)$ is a weighting function for controlling the relative ripples in the passband and stopband. These are typically defined for a lowpass filter as

$$
A(\omega T)= \begin{cases}1, & 0 \leq \omega T \leq \omega_{c} T \\ 0, & \omega_{s} T \leq \omega T \leq \pi\end{cases}
$$

and

$$
W(\omega T)=\left\{\begin{array}{ll}
\delta_{c} / \delta_{s}, & 0 \leq \omega T \leq \omega_{c} T \\
1, & \omega_{s} T \leq \omega T \leq \pi
\end{array},\right.
$$

where $\delta_{c}$ and $\delta_{s}$ are the desired passband and stopband ripples, respectively.

The approximation problem is then stated for a number of angles (frequencies) on a dense grid in the passband and the stopband as [9], [11]

minimize $\delta$

$$
\begin{aligned}
\text { subject to } H_{R}(\omega T)-\frac{\delta}{W\left(\omega_{i} T\right)} & \leq A\left(\omega_{i} T\right), \forall i \\
H_{R}(\omega T)+\frac{\delta}{W\left(\omega_{i} T\right)} & \geq A\left(\omega_{i} T\right), \forall i .
\end{aligned}
$$




\section{B. Joint Linear Programming Design of Coefficient Decima- tion FIR Filters}

For the coefficient decimation approach all different configurations should be considered in the optimization. For this, a minimax optimization based linear programming design problem similar to (12)-(14) is formulated. The constraints are formed assuming a model filter of order $N$, where $N$ is even.

It should be noted that the interpolation of impulse response with a factor $M$ will only cause that the frequency response becomes periodic with a factor $2 \pi / M$. Therefore, we do not need to consider different values of $M$ in the filter design. Instead, we formulate the optimization problem for $M=1$.

Let us introduce the zero-phase transfer function for a filter whose frequency response is decimated with a factor of $D$ into an even-order linear-phase FIR filter as

$$
H_{R, D}(\omega T)=D \sum_{k=0}^{K / D} h(D k) \operatorname{trig}(k, \omega T)
$$

for both odd- and even $D$. Alternatively, for even $D$ there is an alternative decimation that produce an odd-order ${ }^{2}$ linear-phase FIR filter as

$$
H_{R, D}(\omega T)=D \sum_{k=0}^{\left\lfloor\frac{2 K-D}{2 D}\right\rfloor} h(D k+D / 2) \operatorname{trig}(k, \omega T) .
$$

The additional factors $D$ in front of the sum in (15) and (16) are caused by the fact that the decimated impulse response will have a passband gain of $1 / D$ instead of the desired 1 . For a practical implementation this will only shift the signal levels, but it must be considered in the design.

The desired function is dependent on $D$ as

$$
A_{D}\left(\omega_{i} T\right)=\left\{\begin{array}{ll}
1, & 0 \leq \omega_{i} T \leq D \omega_{c} T \\
0, & D \omega_{s} T \leq \omega_{i} T \leq \pi
\end{array} .\right.
$$

The proposed formulation for linear programming design of coefficient decimation FIR filters can then be written as

$$
\begin{aligned}
\operatorname{minimize} & \delta \\
\text { subject to } & H_{R, D}(\omega T)-\frac{\delta}{W\left(\omega_{i} T\right)} \leq A_{D}\left(\omega_{i} T\right), \forall i, D(19) \\
& H_{R, D}(\omega T)+\frac{\delta}{W\left(\omega_{i} T\right)} \geq A_{D}\left(\omega_{i} T\right), \forall i, D .(20)
\end{aligned}
$$

The resulting linear optimization problem in (18)-(20) can then be straightforwardly solved using a standard linear programming solver. Note that all the zero-phase frequency responses, $H_{R, D}$, are using the same set of coefficients.

It should be noted that most previously proposed techniques for linear programming-based design of FIR filters with additional requirements can be applied to the formulation above, although the actual usefulness in the coefficient decimation context must be evaluated from case to case. Examples include constraints on zero coefficient values for Nyquist and $N$ th band filters [12]; fixed-point coefficients [13], [14]; sparseness [15], [16]; flatness constraints [17]; signed-power-of-two terms [18]; and total number of additions in a shift-and-add realization [19].

\footnotetext{
${ }^{2}$ Filter order $2\left(\left\lfloor\frac{2 K-D}{2 D}\right\rfloor\right)+1=2\left(\left\lfloor\frac{N-D}{2 D}\right\rfloor\right)+1$ for $N=2 K \geq D$.
}

TABLE I

Maximum Approximation ERrors For DifFERENT $D_{\max }$.

\begin{tabular}{|c|c|c|}
\hline$D_{\max }$ & Proposed & Conventional \\
\hline 1 & $-55.97 \mathrm{~dB}$ & $-55.97 \mathrm{~dB}$ \\
2 & $-55.50 \mathrm{~dB}$ & $-47.30 \mathrm{~dB}$ \\
3 & $-55.37 \mathrm{~dB}$ & $-47.03 \mathrm{~dB}$ \\
4 & $-55.27 \mathrm{~dB}$ & $-45.25 \mathrm{~dB}$ \\
\hline
\end{tabular}

\section{Design Examples}

Two design examples are presented here to compare the proposed methodology with the previously used approach of optimizing the model filter for the $D=1$ specification only. In this brief, GLPK [20] was used for modeling and solving the optimization problem. The models can be obtained from the authors by request.

\section{A. Design Example 1}

The first example is adapted from [1]. Consider a model filter of order 120 with passband and stopband edges of $\omega_{c} T=$ $0.1 \pi \mathrm{rad}$ and $\omega_{s} T=0.15 \pi \mathrm{rad}$. The weighting factors are selected as $W=1$ in both the passband and the stopband. In addition, we have $D_{\max }=4$. For $D=2,3$, and 4 , the effective filter order is 60,40 , and 30 , respectively. Note that $D=2,3$, and 4 are all integer multiples of the model filter order.

The magnitude responses are shown in Fig. 2 for both the proposed design methodology as well as the conventional method used in previous works. As seen, the proposed method produces filters with lower approximation errors for $D=2,3$, and 4 . The worst case approximation error for the conventional design is $-45.25 \mathrm{~dB}$, while for the proposed method it is $-55.27 \mathrm{~dB}$. For $D=1$ it is slightly increased as the approximation error for the conventional design is $-55.97 \mathrm{~dB}$ for the $D=1$ filter.

It can be noted that the resulting approximations are not equiripple. This is the reason that the otherwise commonly used Parks-McClellan algorithm [8] can not be modified to take several different filter configurations into account, as the convergence of the underlying Remez' exchange algorithm [21] relies on the fact that the maximum absolute value of the error function should be the same for each lobe.

The reason that the approximation error is larger for $D=1$ are the additional design constraints for $D=2,3$, and 4 compared to the conventional design. The more different values of $D$ that are designed for, the larger the approximation error. With more constraints, which can be easily seen from the optimization formulation in the previous section being introduced for each additional $D$, the optimal result can never improve. To further illustrate this effect, let us consider designs with different $D_{\max }$. The resulting approximation errors are shown in Table I. Here it can be seen that the worst approximation error increases with increasing $D_{\max }$. For $D_{\max }=1$ the two designs are, as expected since both are optimal results in the minimax sense for the same constraints, identical.

For the considered design example there are two different ways to decimate the model filter for $D=2$ and 4 . The different decimation alternatives will form optimization constraints 
(a)

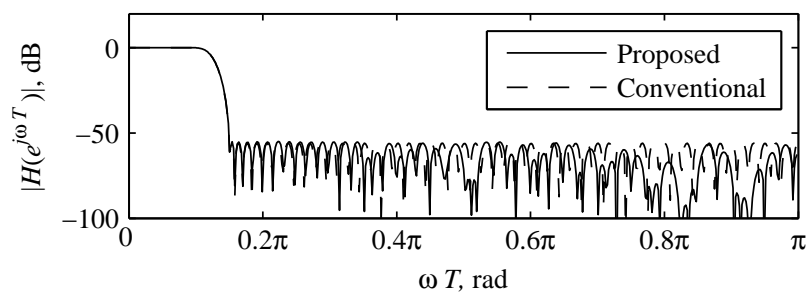

(b)

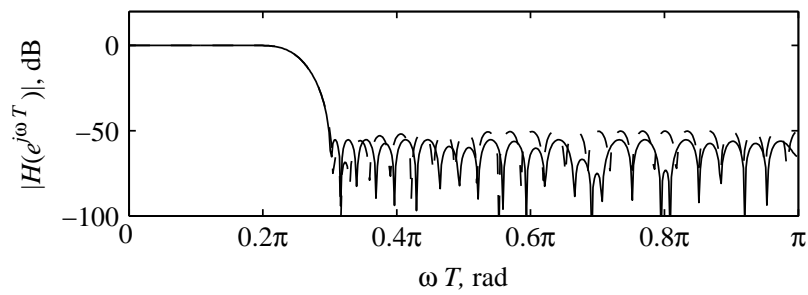

(c)

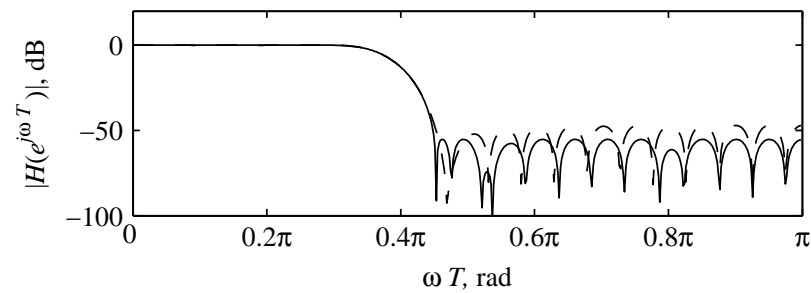

(d)

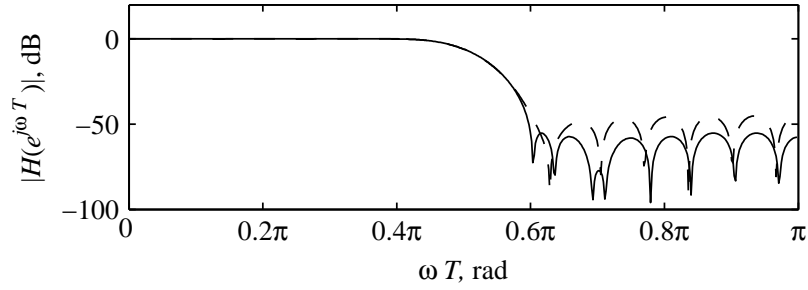

Fig. 2. Magnitude responses for Example 1: (a) $D=1$, (b) $D=2$, (c) $D=3$, and (d) $D=4$.

TABLE II

MAXIMUM APPROXIMATION ERROR FOR DIFFERENT COMBINATIONS OF FILTER ORDERS.

\begin{tabular}{|c|c|c|}
\hline$D=2$ & $D=4$ & Approximation error \\
\hline 60 & 30 & $-55.27 \mathrm{~dB}$ \\
60 & 29 & $-53.72 \mathrm{~dB}$ \\
59 & 30 & $-54.65 \mathrm{~dB}$ \\
59 & 29 & $-53.98 \mathrm{~dB}$ \\
\hline
\end{tabular}

with different variables, and, hence, most likely different approximation errors are obtained. In Table II the different approximation errors for the considered example are shown. Here, it is clear that the decimation used earlier, yielding evenorder filters, is the most advantageous. It could be argued that this is because they have the highest filter orders. However, it can be noted that the 60 and 29 combination gives a larger approximation error compared to the 59 and 29 combination. Hence, there may be specifications where it is worthwhile to consider different decimation cases. (a)

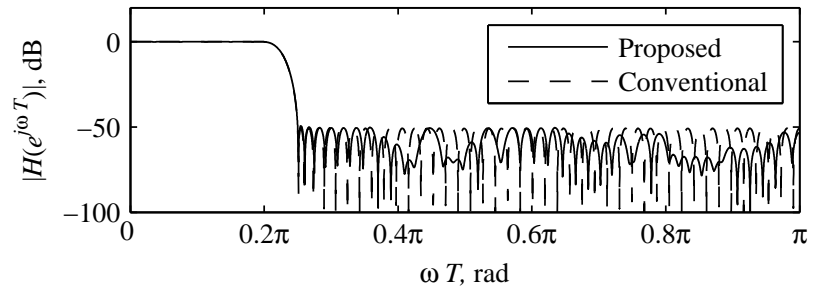

(b)

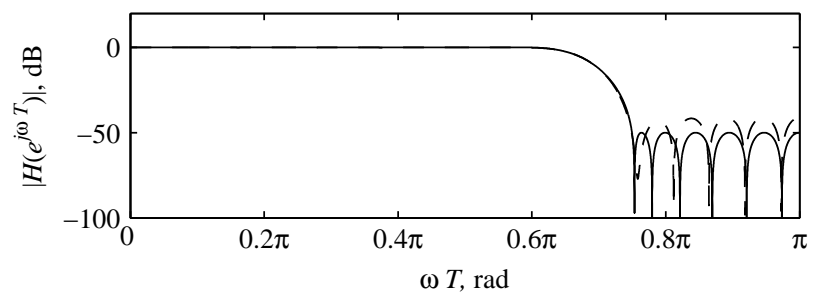

Fig. 3. Magnitude responses for Example 2: (a) CDMA case, $D=1$ and (b) WCDMA case, $D=3$.

\section{B. Design Example 2}

In the second example, a dual standard channel filter for Code Division Multiple Access (CDMA) and Wideband CDMA (WCDMA) described in [6] is used. The overall filter structure consists of a model filter and a masking filter with the purpose of extracting the desired band for channel adaptation. As in [6] we use a 108:th-order model filter with $\omega_{c} T=0.2 \pi \mathrm{rad}$ and $\omega_{s} T=0.25 \pi \mathrm{rad}$. The decimation and interpolation factors are $D=1$ and $M=3$ for the CDMA case and $D=3$ and $M=2$ for the WCDMA case. Unity weights are used for the passband and stopband.

The magnitude responses for the two filters using the proposed method and the conventional method are shown in Fig. 3. Again, the proposed design method produce a significantly lower approximation error of $-49.95 \mathrm{~dB}$ compared to $-41.93 \mathrm{~dB}$ for the conventional.

The design in Fig. 3 is performed with $D=1,2$, and 3 . However, if it is known that the $D=2$ case is not used, it is possible to skip the corresponding constraints. This leads to a slightly smaller approximation error of $-50.28 \mathrm{~dB}$.

\section{COnClusions}

In this brief, a linear programming formulation was proposed for reconfigurable FIR filters based on the coefficient decimation technique. The formulation simultaneously minimizes the largest approximation error in any configuration mode. It was shown that the proposed technique provided significantly better results compared to the previously used design method.

Furthermore, some properties of the coefficient decimation filter approximation error were illustrated. It directly follows from the model and results that the design for more simultaneously optimized configurations implies more constraints in the approximation and, therefore, larger approximation error. Also, it was observed that for even decimation factors there are two possible ways to obtain the decimated impulse response and the effect of this was numerically illustrated. The results show 
that there may be specifications where different decimation cases should be considered.

\section{REFERENCES}

[1] R. Mahesh and A. P. Vinod, "Coefficient decimation approach for realizing reconfigurable finite impulse response filters," in Proc. IEEE Int. Symp. Circuits Syst, Seattle, May 2008, pp. 81-84.

[2] _ - "Reconfigurable frequency response masking filters for software radio channelization," IEEE Trans. Circuits Syst. II, vol. 2, no. 55, pp. 274-278, 2008.

[3] — - "Low complexity flexible filter banks for uniform and non-uniform channelisation in software radios using coefficient decimation," IET Circuits, Devices, Syst., vol. 5, no. 3, pp. 232-242, 2011.

[4] R. Mahesh, A. P. Vinod, E. M.-K. Lai, and A. Omondi, "Filter bank channelizers for multi-standard software defined radio receivers," $J$. Signal Processing Syst., vol. 62, no. 2, pp. 157-171, 2011.

[5] M. Lin, A. P. Vinod, and C. M. S. See, "A new flexible filter bank for low complexity spectrum sensing in cognitive radios," J. Signal Processing Syst., vol. 62, no. 2, pp. 205-215, 2011.

[6] K. G. Smitha and A. P. Vinod, "A new low power reconfigurable decimation-interpolation and masking based filter architecture for channel adaptation in cognitive radio handsets," Physical Commun., vol. 2, no. 4, pp. 47-57, 2009.

[7] Y. C. Lim, "Frequency-response masking approach for the synthesis of sharp linear phase digital filters," IEEE Trans. Circuits Syst., vol. CAS33, no. 4, pp. 357-364, Apr. 1986.

[8] T. Parks and J. McClellan, "Chebyshev approximation for nonrecursive digital filters with linear phase," IEEE Trans. Circuit Theory, vol. 19, no. 2, pp. 189-194, 1972.

[9] L. Wanhammar and H. Johansson, Digital Filters. Department of Electrical Engineering, Linköping University, 2011.

[10] R. A. Hawley, B. C. Wong, J. Laskowski, and H. Samueli, "Design techniques for silicon compiler implementations of high speed FIR digital filters," IEEE J. Solid-State Circuits, vol. 31, no. 5, pp. 656666, 1996.

[11] L. Rabiner, "Linear program design of finite impulse response (FIR) digital filters," IEEE Trans. Audio Electroacoust., vol. AU-20, pp. 280 288, Oct. 1972.

[12] J.-K. Liang, R. de Figueiredo, and F. Lu, "Design of optimal Nyquist, partial response, Nth band, and nonuniform tap spacing FIR digital filters using linear programming techniques," IEEE Trans. Circuits Syst., vol. 32, no. 4, pp. 386-392, 1985.

[13] D. Kodek, "Design of optimal finite wordlength FIR digital filters using integer programming techniques," IEEE Trans. Acoust., Speech, Signal Process., vol. 28, no. 3, pp. 304-308, 1980.

[14] Y. C. Lim, "Design of discrete-coefficient-value linear phase FIR filters with optimum normalized peak ripple magnitude," IEEE Trans. Circuits Syst., vol. 37, no. 12, pp. 1480-1486, Dec. 1990.

[15] O. Gustafsson, L. S. Debrunner, V. DeBrunner, and H. Johansson, "On the design of sparse half-band like FIR filters," in Proc. Asilomar Conf. Signals Systems Computers, Nov. 2007, pp. 1098-1102.

[16] T. Baran, D. Wei, and A. V. Oppenheim, "Linear programming algorithms for sparse filter design," IEEE Trans. Signal Process., vol. 58, no. 3, pp. 1605-1617, 2010.

[17] K. Steiglitz, T. W. Parks, and J. F. Kaiser, "METEOR: a constraint-based FIR filter design program," IEEE Trans. Signal Process., vol. 40, no. 8, pp. 1901-1909, 1992.

[18] O. Gustafsson, H. Johansson, and L. Wanhammar, "An MILP approach for the design of linear-phase FIR filters with minimum number of signed-power of two terms," in Proc. European Conf. Circuit Theory Design, Espoo, Finland, Aug. 2001.

[19] D. Shi and Y. J. Yu, "Design of linear phase FIR filters with high probability of achieving minimum number of adders," IEEE Trans. Circuits Syst. I, vol. 58, no. 1, pp. 126-136, 2011.

[20] GNU Linear Programming Kit 4.46, http://www.gnu.org/software/glpk/, 2011.

[21] E. Ya. Remez, General computational methods of Chebyshev approximation: The problems with linear real parameters. Washington, DC: Atomic Energy Commission Translation Series, 1957, vol. 4491. 